\title{
Depression Risk Factors and Affect Dynamics: An Experience Sampling Study
}

\author{
Carter J. Funkhouser, ${ }^{\text {a,b }}$ Ariela J. E. Kaiser, ${ }^{b}$ Kira L. Alqueza, ${ }^{c}$ Vivian L. Carrillo, ${ }^{a}$ Lija M. K. \\ Hoffman, ${ }^{a}$ Carver B. Nabb, ${ }^{a}$ Randy P. Auerbach, ${ }^{\mathrm{c}, \mathrm{d}}$ \& Stewart A. Shankman ${ }^{\mathrm{a}}$ \\ ${ }^{a}$ Northwestern University \\ Department of Psychiatry and Behavioral Sciences \\ 680 N. Lake Shore Drive, Chicago, IL 60611 \\ ${ }^{\mathrm{b}}$ University of Illinois at Chicago \\ Department of Psychology \\ 1007 W. Harrison Street, Chicago, IL 60607 \\ ${ }^{\mathrm{c} C}$ Columbia University \\ Department of Psychiatry \\ 1051 Riverside Drive, New York, NY 10032 \\ ${ }^{\mathrm{d}}$ Sackler Institute \\ Division of Clinical Developmental Neuroscience \\ 1051 Riverside Drive, New York, NY 10032
}

This manuscript has been accepted for publication in Journal of Psychiatric Research. It will undergo copyediting, typesetting, and review of the resulting proof before being published in its final form.

Declarations of interest: none

Funding: This work was supported by the National Institute of Mental Health [grants R21MH112330 and R01MH119771]. The content is solely the responsibility of the authors and does not necessarily represent the official views of the National Institutes of Health or of any other sponsor.

Correspondence concerning this article should be addressed to Stewart A. Shankman, Department of Psychiatry and Behavioral Sciences, Northwestern University, Chicago, IL, 60611. Email: stew.shankman@,northwestern.edu 


\begin{abstract}
Affect dynamics reflect individual differences in how emotional information is processed, and may provide insights into how depressive episodes develop. To extend prior studies that examined affect dynamics in currently depressed individuals, the present study tested in 68 nondepressed young adults whether three well-established risk factors for major depressive disorder (MDD) - (a) past episodes of MDD, (b) family history of MDD, and (c) reduced neurophysiological responses to reward - predicted mean levels, instability, or inertia (i.e., inflexibility) of positive affect (PA) and/or negative affect (NA). Momentary PA and NA were assessed up to 6 times per day for 14 days (mean number of surveys completed=45.89). MDD history and family history of MDD were assessed via semi-structured interview, and neurophysiological responses to reward were indexed using the Reward Positivity, an eventrelated potential related to depression. After adjusting for current depressive symptoms, results indicated that (a) past episodes of MDD predicted higher mean levels of NA, (b) family history of MDD predicted greater PA inertia, and (c) blunted reactivity to reward predicted greater NA inertia. Collectively, these results suggest that elevated mean levels of NA and inflexibility of PA and NA may be potential mechanisms that confer risk for depression.

Keywords: Major Depressive Disorder; Emotion; Inertia; Instability; Reward Positivity (RewP); Family History
\end{abstract}




\section{Introduction}

Major Depressive Disorder (MDD) is one of the most prevalent and disabling mental disorders (Friedrich, 2017; Kessler et al., 2005). Efforts to identify indicators of MDD risk have highlighted several factors that are strongly and consistently associated with MDD. First, a personal history of MDD is associated with a $69 \%$ increase in risk for future depressive episodes (Buckman et al., 2018). Second, individuals with a family history of MDD are three to five times more likely to develop MDD (Klein et al., 2004; Weissman et al., 2006). Third, deficits in how individuals process reward are consistently linked to MDD morbidity and risk. Specifically, a blunted Reward Positivity (RewP), an event-related potential (ERP) reflecting reduced reward responsiveness, predicted (a) a greater likelihood of developing MDD in the future (Nelson et al., 2016), (b) greater familial MDD risk (Weinberg et al., 2015), and (c) poorer treatment response (Burkhouse et al., 2016). In sum, MDD history, family history of MDD, and reward processing deficits are robust, widely studied indicators of risk for depression onset or recurrence.

An increasingly popular ontological perspective posits that MDD represents a dynamic system reflecting the continuous interplay of moment-to-moment emotions, cognitions, and behaviors (Wichers, 2014). Studies adopting this view have often used experience sampling methodology (ESM), which involves the repeated collection of data over time to assess realtime, ecologically valid momentary experiences (Solhan et al., 2009). Low positive affect (PA) and high negative affect (NA) are theorized to be central features of MDD (Clark and Watson, 1991), and ESM studies have consistently shown that individuals with MDD experience lower mean levels of PA and higher mean levels of NA than healthy controls (Aan het Rot et al., 2012). In addition to examining mean PA and NA, depression studies have increasingly examined dynamics in affective experience. Affect dynamics refer to a set of processes involving the 
temporal dynamics of emotional states (Trull et al., 2015). Two indicators of affect dynamics likely to be particularly relevant to MDD are instability, or the magnitude of moment-to-moment fluctuations in affect, ${ }^{1}$ and inertia, or the extent to which affect is resistant to change or persists over time. A meta-analysis suggested that depressive symptoms are associated with increased instability and inertia of both PA and NA (Houben et al., 2015), and there is preliminary evidence showing that instability and inertia of PA and NA may be disrupted prior to MDD onset (Kuppens et al., 2012) and in remission (Schoevers et al., 2020). Changes in affect dynamics may reflect early warning signs that precede transitions between normal and depressed mood states (van de Leemput et al., 2014; Wichers, 2014), further supporting the potential utility of affect dynamics in predicting treatment response (Husen et al., 2016) and personalizing interventions (Fisher and Boswell, 2016).

Although affect dynamics may reflect important processes involved in the exacerbation of depressive symptoms, few studies have examined associations between affect dynamics and depression risk factors. Comparisons of depressed and non-depressed individuals are unable to distinguish between risk factors that precede depressive episodes and concomitants of a depressive episode (Kraemer et al., 1997). Examining individual differences in non-depressed individuals at various levels of risk rules out the possibility that an observed association is merely a concomitant of a depressive episode, which is important for clarifying predictors of depression onset or recurrence, identifying potential mechanisms implicated in the pathophysiology of depression, and preventing depressive episodes. Therefore, the present study tested whether three well-established risk factors for MDD-lifetime history, family history, and

\footnotetext{
${ }^{1}$ Some studies have also examined affect variability (i.e., the within-person standard deviation). Instability captures dynamic affective processes better than variability because the latter does not consider temporal dependency (i.e., affect at time $t$ occurring before affect at time $t+1$ ) and only reflects dispersion from one's mean level.
} 
reduced neurophysiological reward sensitivity-predicted mean levels, instability, or inertia of PA and NA in a non-depressed sample. Based on the theorized relationship between depression and mean levels of PA and NA (Clark and Watson, 1991; Shankman and Klein, 2003), as well as findings from previous ESM studies in depressed individuals (Aan het Rot et al., 2012; Houben et al., 2015), it was hypothesized that the three risk factors would predict greater mean NA, lower mean PA, and greater PA and NA instability and inertia.

\section{Materials and Methods}

\section{Participants}

Participants were non-depressed young adults, all of whom had previously participated in a parent study investigating familial risk for internalizing psychopathology (Stevens et al., 2019; Weinberg et al., 2016). The parent sample primarily consisted of sibling pairs aged 18-30 (to ensure they were in the peak risk window for internalizing psychopathology; Kessler et al., 2005) recruited from the community. Individuals in the parent sample who had consented to receive communications regarding additional study participation opportunities were contacted via phone and/or email. To maximize sample size, potential participants were recruited for the present study regardless of whether their sibling could participate. Potential participants then completed a phone screen to assess inclusion and exclusion criteria. Although the parent study had no diagnosis-based inclusion or exclusion criteria, the present study excluded potential participants if they met diagnostic criteria for current MDD due to the focus on risk for depression. Sixtyeight individuals participated in the present study, but nine participants with fewer than ten completed ESM surveys were excluded from all analyses, leaving 59 individuals (49 singletons and five sibling pairs). An additional seven participants were excluded from analyses of affect inertia due to having too few $(<10)$ pairs of consecutive completed ESM surveys. These 
exclusion criteria were chosen to maximize the reliability of parameter estimates while retaining a sufficiently large sample for analyses. Study procedures were approved by the university institutional review board, and informed consent was obtained from all participants.

Demographic and clinical characteristics are summarized in Table 1.

\section{Instruments}

Structured Clinical Interview for DSM-5. Lifetime and current MDD and other diagnoses were assessed using the Structured Clinical Interview for DSM-5 (SCID; First et al., 2015). Lifetime MDD diagnosis had moderate test-retest reliability $(\mathrm{k}=.69)$ in a subsample of the larger parent study (Shankman et al., 2018). As part of the parent study, as many first-degree relatives as possible $(M=2.83$ relatives per participant, $S D=.87)$ completed the SCID in person or via phone to assess lifetime MDD and other disorders. MDD family history was defined dichotomously as having one or more interviewed relatives with lifetime MDD.

Inventory of Depression and Anxiety Symptoms-II. The twenty-item General Depression scale from the Inventory of Depression and Anxiety Symptoms-II (IDAS; Watson et al., 2012) was used to measure depressive symptom severity over the past two weeks. The General Depression scale had excellent internal consistency in the present study $(\alpha=.88)$, and has demonstrated strong psychometric properties (Watson et al., 2012, 2008).

\section{Reward Positivity Task}

The RewP was elicited using the Doors Task (Foti and Hajcak, 2010), a simple gambling task in which participants can either gain or lose money. The Doors Task was modified in the present study to include a neutral feedback option in which no money was gained or lost (i.e., 'breaking even'). At the beginning of each trial, participants were presented with an image of three doors and were instructed to choose one door by pressing the corresponding button on a 
keyboard. The doors remained on the screen until the participant responded. After the response, a fixation cross appeared for 1,000 ms and feedback was presented on the screen for 2,000 ms. Feedback consisted of either a green " $\uparrow "$ (indicating a gain of \$.80), a red " $\downarrow$ " (indicating a loss of \$.40), or a yellow “_”" (indicating no money was gained or lost). The amounts of monetary gain and loss were selected to give gain and loss equally subjective values (Tversky and Kahneman, 1992). Next, a fixation mark appeared for 1,500 ms and was followed by the message "Click for the next round," which remained on the screen until the participant responded, thus beginning the next trial. The task consisted of 30 gain, loss, and neutral trials (90 trials total) presented in a pseudorandom order.

Continuous electroencephalography (EEG) data were recorded using an elastic cap and the ActiveTwo BioSemi system (BioSemi, Amsterdam, Netherlands). Sixty-four Ag/AgCl electrodes were used, based on the 10-20 system, as well as two electrodes on the right and left mastoids. The electrooculogram (EOG) generated from eye movements was recorded using four facial electrodes. Horizontal eye movements were measured using two electrodes located approximately $1 \mathrm{~cm}$ outside the outer edge of the right and left eyes, and vertical eye movements and eyeblinks were measured via one electrode placed approximately $1 \mathrm{~cm}$ below the left eye and electrode FP1. The data were digitized at a sampling rate of 1,024 Hz, using a low-pass fifth order sinc filter with $-3 \mathrm{~dB}$ cutoff point at $208 \mathrm{~Hz}$. Each active electrode was measured online with respect to a common mode sense (CMS) active electrode, located between PO3 and POz, producing a monopolar (nondifferential) channel. CMS forms a feedback loop with a paired driven right leg (DRL) electrode located between POz and PO4, reducing the potential and increasing the common mode rejection rate. 
Offline, all data were analyzed in BrainVision Analyzer 2.1.0 (Brain Products, Munich, Germany), and were referenced to the average of the left and right mastoids and band-pass filtered with low and high cutoffs of 0.1 and $30 \mathrm{~Hz}$, respectively. Eye blink and ocular corrections were conducted using established standards (Gratton et al., 1983). EEG channels with a high number of channel-specific artifacts were removed and interpolated (spline interpolation; Perrin et al., 1989). The mean number of interpolated channels was 1.71 (maximum=7). EEG data were segmented into epochs beginning $200 \mathrm{~ms}$ before feedback onset and ending 1,000 ms after feedback onset. The $200 \mathrm{~ms}$ prior to feedback onset was used for baseline correction. Epochs for individual channels were rejected using a semi-automated procedure, with artifacts identified using the following criteria: a voltage step of more than 50 $\mu \mathrm{V}$ between sample points, a voltage difference of $300 \mu \mathrm{V}$ within a trial, and a maximum voltage difference of less than $50 \mu \mathrm{V}$ within $100 \mathrm{~ms}$ intervals. These intervals were rejected from individual channels in each trial. Visual inspection of the data was then conducted to detect and reject remaining artifacts. After interpolation and artifact rejection, the average number of trials retained for averaging was $29.6,29.5$, and 29.6 for the gain, loss, and neutral conditions, respectively. The RewP was scored as the mean amplitude from 250-350 ms following feedback at a pooling of $\mathrm{FCz}$ and $\mathrm{Cz}$, where the difference between gain and non-gain amplitudes was maximal (see Figure 1). Consistent with recommendations for isolating ERPs to activity specific to one condition (Meyer et al., 2017), responses to loss and neutral trials were regressed on responses to gain trials, thus creating a residual score for the RewP. Split-half reliabilities for each condition were calculated using the correlation between the averages of odd- and evennumbered trials corrected using the Spearman-Brown prophecy formula (Nunnally and 
Bernstein, 1994), and were $.94, .91$, and .89 for the gain, loss, and neutral conditions, respectively.

\section{Experience Sampling Methodology}

Following the baseline assessment, participants received ESM prompts six times daily at random times within stratified 90-minute windows between 8:30am and 10:30pm for 14 consecutive days. During each prompt, participants received the 10-item Positive and Negative Affect Schedule-Short Form (PANAS-SF; Kercher, 1992). Positive affect (PA; excited, enthusiastic, alert, inspired, and determined) and negative affect (NA; distressed, upset, scared, nervous, and afraid) items were rated on a 5-point Likert scale ranging from 1 (very slightly or not at all) to 5 (extremely), and item order was randomized each day. Participants had twenty minutes to complete the ESM protocol, after which it was no longer available. Participants completed an average of 45.89 surveys over the ESM period, corresponding to a compliance rate of $54.6 \%$. As affect dynamics indices may differ depending on the time lag examined (Gollob and Reichardt, 1987), we excluded longer time lags in analyses of instability and inertia. Instability analyses excluded lags greater than 1.5 standard deviations above the sample mean (resulting in an average lag of 172.33 minutes; range $=54.88-354.12$ ), and inertia analyses focused on surveys completed within successive windows (mean lag $=149.46$ minutes; range $=$

54.88-247.55). The 52 participants included in analyses of affective inertia had an average of 28.46 pairs of consecutive completed surveys $(S D=12.23$; range $=11-58)$

\section{Procedures}

At a baseline assessment, participants were administered the SCID by trained study staff, completed the self-reported IDAS, and the Doors Task while EEG data were recorded. Following EEG acquisition, the Metricwire (Metricwire Inc., Kitchener, Canada) app was 
installed on participants' smartphone, and participants were instructed on how to complete the ESM items. Participants also were reminded that EMS prompts would occur 6 times per day over the course of 14 days, and participants were called with reminders to complete surveys during that time.

\section{Data Analysis}

As ESM observations (level 1) are nested within individuals (level 2) ${ }^{2}$, multilevel modeling was used to estimate relationships between each MDD risk factor and mean, instability, and inertia of PA or NA. All models included a random intercept to allow mean levels of PA or NA to vary freely across individuals. Models also covaried for current depressive symptom severity (IDAS General Depression scale), as subthreshold depression has been shown to be associated with mean levels, instability, and inertia of affect (Houben et al., 2015). This additionally allowed tests of the predictive power of the three risk factors independent of residual current depressive symptoms. To examine relationships between MDD risk factors and mean levels of momentary PA and NA, separate multilevel models were estimated for each risk factor as a fixed effect predictor of PA or NA. Significant relationships between a risk factor and mean PA or NA were followed up with a model testing whether affect valence moderated the relationship between the risk factor and mean affect levels. Significant moderating effects of affect valence would indicate that the risk factor is differentially associated with mean levels of PA versus NA.

Affect instability was operationalized using adjusted square of successive differences (ASSDs) following Jahng et al. (2008). Successive differences (i.e., affect $t_{t+1}-$ affect $_{t}$ ) were first

\footnotetext{
${ }^{2}$ The present study included five sibling pairs ( 3 individuals with remitted MDD, 7 healthy controls). As $91 \%$ of families contained one participant, family effects could not be distinguished from person-level effects and thus were not included.
} 
calculated separately for PA and NA, and were then adjusted by dividing each successive difference by $\left[\left(t_{i+1}-t_{\mathrm{i}}\right) / \text { median }\left(t_{\mathrm{i}+1}-t_{\mathrm{i}}\right)\right]^{\lambda}$. Lambda $(\lambda)$ was calculated separately for PA and NA, and was chosen to make the successive difference as constant as possible across participants. Adjusted successive differences were then squared. Separate multilevel models predicting ASSDs (i.e., affect instability) were estimated for each MDD risk factor and for PA and NA. As recent studies have questioned the incremental validity of affect instability over mean affect (Dejonckheere et al., 2019), instability analyses also controlled for mean affect.

Affect inertia was analyzed using multilevel autoregressive models in which affect $t_{t}$ predicted affect $t_{++1}$. The lagged level 1 predictor $\left(\right.$ affect $\left._{t}\right)$ was within-person centered for inertia analyses to allow for an interpretation of within-person effects (Hamaker and Grasman, 2014), and the autoregressive relationship was modeled as a random slope to represent individual differences in inertia. Inertia models controlled for the linear effect of time because previous studies have reported linear time trends in ESM data (e.g., Koval et al., 2015a). To determine if inertia was moderated by any of the MDD risk factors, we estimated separate models for each risk factor and for PA and NA, each of which included a cross-level interaction between the level 1 autoregressive slope and the level 2 risk factor. As a result, the level 1 intercept reflected each person's mean level of PA or NA across all observations. Between-day (i.e., overnight) lags were excluded from computations of instability and inertia by setting the first affect ratings of the day to missing when the last ESM survey on the preceding day was non-missing.

All models were estimated via maximum likelihood, and semi-partial $\mathrm{R}^{2}$ for fixed effects were calculated using Nakagawa and Schielzeth (2013) guidelines. Analyses were performed in R (R Core Team, 2019) using the lme4 (Bates et al., 2015) and lmerTest (Kuznetsova et al., 2017) packages. 


\section{Results}

\section{Preliminary Analyses}

Similar to previous studies (Funkhouser et al., 2019; Weinberg and Shankman, 2017), ERPs to feedback in the Doors Task differed as a function of condition, $F(2,114)=44.94$, $p<.001, \mathrm{R}^{2}=.12$, such that mean amplitudes were significantly greater following monetary gain relative to both loss, $t(114)=4.18, p<.001$, semi-partial $\mathrm{R}^{2}=.03$, and neutral feedback, $t(114)=9.46, p<.001$, semi-partial $\mathrm{R}^{2}=.12$. Amplitudes were more positive following monetary loss than neutral feedback, $t(114)=5.28, p<.001$, semi-partial $\mathrm{R}^{2}=.04$.

\section{Depression Risk Factors and ESM}

Correlations between continuous study variables are presented in Figure 3. The three MDD risk factors were not associated with each other $(p s>.05)$. Participants reported an average IDAS General Depression score of $32.92(S D=8.86)$, which corresponds to the $44^{\text {th }}$ percentile in a national norming sample (Nelson et al., 2018). Depression severity was greater in individuals with a history of $\operatorname{MDD}(M=37.40, S D=9.44)$ compared to healthy controls $(M=28.28, S D=5.14)$, $t(45.12)=4.63, p<.001, d=1.22$, but did not differ between participants with and without a family history of MDD, $t(40.75)=1.39, p=.173, d=.39$, and was unrelated to the RewP, $r=-.12, t(57)=.91$, $p=.367$. ESM compliance was unrelated to any of the depression risk factors or current depressive symptom severity $(p \mathrm{~s}>.05)$. Multilevel models indicated that, with the exception of a significant negative association between the number of completed surveys and PA instability, $\beta=-.10, t(53.06)=2.51, p=.015$, semi-partial $\mathrm{R}^{2}=.01$, neither ESM compliance nor current depressive symptom severity were significantly related to mean, instability, or inertia of PA or NA $(p s>.05)$. Consistent with theoretical accounts of PA and NA as relatively orthogonal (e.g., Watson et al., 1988), PA and NA were unrelated within individuals $(p>.05)$. 
Results of multilevel models predicting mean, instability, or inertia of PA or NA while covarying for current depression symptom severity are summarized in Table 2. MDD history was significantly related to mean NA, $\beta=.42, t(59.86)=2.48, p=.016$, semi-partial $\mathrm{R}^{2}=.03$, as individuals with a history of MDD had higher mean levels of NA than healthy controls. This effect remained significant in a model that included the other two risk factors as additional predictors, $\beta=.40, t(56.92)=2.42, p=.019$, semi-partial $\mathrm{R}^{2}=.03$. To test whether this effect was specific to (or stronger for) NA, we tested whether affect valence (PA versus NA) moderated the relationship between MDD history and momentary affect while controlling for depressive symptom severity and the other two depression risk factors. Affect valence significantly moderated this relationship, $\beta=.61, t(5356.71)=15.46, p<.001$, semi-partial $\mathrm{R}^{2}=.03$, such that MDD history was more strongly related to mean levels of NA than PA. No risk factors were significantly associated with mean PA.

No risk factors were significantly associated with instability of PA or NA. However, participants with a family history of MDD had greater PA inertia independent of current depressive symptom severity, $\beta=.10, t(30.81)=2.48, p=.019$, semi-partial $\mathrm{R}^{2}<.01$, and when the other two risk factors and their interactions with PA at time $t$ were included as additional predictors, $\beta=.09, t(33.78)=2.13, p=.041$, semi-partial $\mathrm{R}^{2}<.01$. Additionally, the RewP negatively predicted NA inertia above and beyond current depression symptoms, $\beta=-.08, t(38.11)=2.04$, $p=.048$, semi-partial $\mathrm{R}^{2}=.01$, indicating that individuals with less neural reactivity to reward had greater persistence of NA from one time point to the next. However, the effect for RewP was weaker and non-significant when the other two risk factors and their interactions with NA at time $t$ were added as additional covariates, $\beta=-.04, t(53.52)=.96, p=.343$, semi-partial $\mathrm{R}^{2}<.01$. Best linear unbiased predictions (BLUPs; Robinson, 1991) for each participant's random 
autoregressive slope of NA (i.e., estimated NA inertia) controlling for current depression symptom severity and the linear effect of time are plotted in relation to the RewP (Figure 2).

\section{Discussion}

The present study tested the relationships between three well-established depression risk factors and different aspects of affect dynamics. Individuals with a past episode of MDD reported greater mean levels of NA than controls, those with a family history of MDD had greater PA inertia, and reduced neural reactivity to reward was associated with greater NA inertia. Taken together, these findings suggest that risk factors for MDD may be distinctly associated with different aspects of affect dynamics.

The finding that individuals with past episodes of MDD experience higher mean levels of NA in their daily lives is consistent with prior research (Aan het Rot et al., 2012; Schoevers et al., 2020) and the theoretical importance of NA in depression (Clark and Watson, 1991). This relationship was independent of current depressive symptom severity and other risk factors, indicating that it was not attributable to residual depression symptoms (in participants with remitted MDD) or shared variance with other risk factors. This relationship was also significantly stronger for NA than PA. In prior studies, elevated mean levels of NA prospectively predicted depression recurrence (Wichers et al., 2010) and thus, may represent a target for preventative interventions. Importantly, studying individuals with remitted MDD precludes the ability to determine whether elevated mean NA preceded (reflecting vulnerability) or was caused by the prior depressive episode (reflecting a 'scar'; Zeiss and Lewinsohn, 1988).

The present study also found that a family history of MDD was associated with greater PA inertia above and beyond current depressive severity, MDD history, and neural reward sensitivity. Stronger affective inertia is thought to reflect resistance or inflexibility to external 
(e.g., events) or internal (e.g., emotion regulation) influences (Hollenstein, 2015), and greater PA and NA inertia have been associated with current depression symptoms and future depression onset (Houben et al., 2015; Kuppens et al., 2012). Component processes of affective inflexibility such as maladaptive emotion regulation strategies (e.g., suppression; Koval et al., 2015b) are implicated in depression (Beblo et al., 2012), and may be mechanisms underlying the relationship between family history of depression and PA inertia.

Additionally, neural reward sensitivity was associated with inertia of NA, indicating that individuals who had less neural reactivity to reward had greater persistence of NA over time. This effect was independent of current depressive symptoms, but nonsignificant and slightly weaker when MDD history and family history were included as additional predictors. It is possible that those with blunted reward sensitivity experience a smaller reduction in NA following positive events relative to those with greater reward sensitivity. Additional work is needed to elucidate (a) how the reward processing system unfolds in daily life, (b) how this system is disrupted in individuals at risk for depression, and, (c) broader associations between laboratory reward measures and real-world affect, affect dynamics, and behavior (Bakker et al., 2017; Waugh et al., 2017).

The relationships between depression risk factors and emotional inertia were small in magnitude, which was expected given that depression itself is only moderately related to affective inertia (Houben et al., 2015). It also is worth considering whether effects related to emotional inertia are specific to one affect valence and/or index of affect dynamics. It is unclear why family history was related to inertia of PA but not NA, and it is somewhat surprising that neural sensitivity to reward was related to inertia of NA but not PA (Ironside et al., 2018). These relationships may reflect distinct mechanisms underlying risk for depression. If so, this would 
suggest that the emotion context insensitivity hypothesis (Rottenberg, 2005), which asserts that depression is characterized by blunted reactivity and flexibility of both positive and negative emotions, may not extend to individuals at risk for depression. However, further research examining the specificity of these relationships is needed, as the use of multilevel modeling precluded analyses testing whether affect valence moderated relationships between risk factors and inertia or whether effects were specific to inertia.

Depression symptom severity was unrelated to mean, instability, or inertia of PA or NA, which is somewhat inconsistent with results from previous studies, including some studies of non-clinically ascertained samples (e.g., Houben et al., 2015; Sperry et al., 2020). There are several differences that may account for these discrepant results. First, individuals with current MDD were excluded from the present study but have generally not been excluded in relevant prior studies of non-clinically ascertained samples. The exclusion of individuals with clinical levels of depression symptom severity likely restricted the upper range of depressive symptom severity, potentially inhibiting the ability to detect these effects. Second, the present study assessed depressive symptoms using the IDAS General Depression scale, whereas prior studies have typically used more widely used measures such as the Beck Depression Inventory. Although the IDAS General Depression scale has demonstrated convergent validity with other depression measures (Watson et al., 2007), differences in item content across measures may have contributed to discrepant findings regarding associations between depression symptom severity and affect dynamics (Fried, 2017).

Strengths of this study include the relatively novel focus of studying risk factors in nondepressed individuals, the inclusion of a neurophysiological measure of reward processing, the measurement of MDD family history through direct interviews with first-degree relatives, and 
the inclusion of current depression symptom severity as a covariate. There are also several important limitations in the present study. First, unmeasured factors such as experiences of and reactivity to positive and negative events, emotion regulation, and sleep may have impacted momentary emotions during the ESM period (Aan het Rot et al., 2012; Pemberton and Fuller Tyszkiewicz, 2016). Second, the sample size and ESM response rate were somewhat lower than in some other affect dynamics studies, which may have decreased statistical power. Third, results may not generalize beyond the depression risk factors or time lags examined in the present study (Hollenstein et al., 2013). Fourth, family history of MDD was likely underestimated because not all first-degree relatives were interviewed, and this underestimation may have influenced the results of the family history analyses.

\section{Conclusion}

The present study tested whether three well-established risk factors for depression were related to momentary affect and affect dynamics. MDD history was associated with higher mean NA, family history of MDD was associated with greater PA inertia, and reduced reward sensitivity was associated with greater NA inertia. These findings suggest that studying realworld affect and affect dynamics can elucidate potential mechanisms underlying risk for depression. 


\section{References}

Aan het Rot, M., Hogenelst, K., Schoevers, R.A., 2012. Mood disorders in everyday life: A systematic review of experience sampling and ecological momentary assessment studies. Clin. Psychol. Rev. 32, 510-523. https://doi.org/10.1016/j.cpr.2012.05.007

Bakker, J.M., Goossens, L., Lange, I., Michielse, S., Schruers, K., Lieverse, R., Marcelis, M., van Amelsvoort, T., van Os, J., Myin-Germeys, I., Wichers, M., 2017. Real-life validation of reduced reward processing in emerging adults with depressive symptoms. J. Abnorm. Psychol. 126, 713-725. https://doi.org/10.1037/abn0000294

Bates, D., Mächler, M., Bolker, B.M., Walker, S.C., 2015. Fitting linear mixed-effects models using lme4. J. Stat. Softw. 67. https://doi.org/10.18637/jss.v067.i01

Beblo, T., Fernando, S., Klocke, S., Griepenstroh, J., Aschenbrenner, S., Driessen, M., 2012. Increased suppression of negative and positive emotions in major depression. J. Affect. Disord. 141, 474-479. https://doi.org/10.1016/j.jad.2012.03.019

Buckman, J.E.J., Underwood, A., Clarke, K., Saunders, R., Hollon, S.D., Fearon, P., Pilling, S., 2018. Risk factors for relapse and recurrence of depression in adults and how they operate: A four-phase systematic review and meta-synthesis. Clin. Psychol. Rev. 64, 13-38. https://doi.org/10.1016/j.cpr.2018.07.005

Burkhouse, K.L., Kujawa, A., Kennedy, A.E., Shankman, S.A., Langenecker, S.A., Phan, K.L., Klumpp, H., 2016. Neural Reactivity to Reward as a Predictor of Cognitive Behavioral Therapy Response in Anxiety and Depression. Depress. Anxiety 33, 281-288. https://doi.org/10.1002/da.22482

Clark, L.A., Watson, D., 1991. Tripartite model of anxiety and depression: Psychometric evidence and taxonomic implications. J. Abnorm. Psychol. 100, 316-36. 
https://doi.org/10.1037/0021-843X.100.3.316

Dejonckheere, E., Mestdagh, M., Houben, M., Rutten, I., Sels, L., Kuppens, P., Tuerlinckx, F., 2019. Complex affect dynamics add limited information to the prediction of psychological well-being. Nat. Hum. Behav. 3, 478-491. https://doi.org/10.1038/s41562-019-0555-0

First, M.B., Williams, J.B.W., Karg, R.S., Spitzer, R.L., 2015. Structured Clinical Interview for DSM-5-Research Version. American Psychiatric Association. https://doi.org/10.1002/9781118625392.wbecp351

Fisher, A.J., Boswell, J.F., 2016. Enhancing the Personalization of Psychotherapy With Dynamic Assessment and Modeling. Assessment 23, 496-506. https://doi.org/10.1177/1073191116638735

Foti, D., Hajcak, G., 2010. State sadness reduces neural sensitivity to nonrewards versus rewards. Neuroreport 21, 143-147. https://doi.org/10.1097/WNR.0b013e3283356448

Fried, E.I., 2017. The 52 symptoms of major depression: Lack of content overlap among seven common depression scales. J. Affect. Disord. https://doi.org/10.1016/j.jad.2016.10.019

Friedrich, M.J., 2017. Depression is the leading cause of disability around the world. JAMA 317 , 1517. https://doi.org/10.1001/jama.2017.3826

Funkhouser, C.J., Auerbach, R.P., Kujawa, A., Morelli, S.A., Phan, K.L., Shankman, S.A., 2019. Social Feedback Valence Differentially Modulates the Reward Positivity, P300, and Late Positive Potential. J. Psychophysiol. https://doi.org/10.1027/0269-8803/a000253

Gollob, H.F., Reichardt, C.S., 1987. Taking account of time lags in causal models. Child Dev. 58, 80. https://doi.org/10.2307/1130293

Gratton, G., Coles, M.G.H., Donchin, E., 1983. A new method for off-line removal of ocular artifact. Electroencephalogr. Clin. Neurophysiol. 55, 468-484. 
https://doi.org/10.1016/0013-4694(83)90135-9

Hamaker, E.L., Grasman, R.P.P.P., 2014. To center or not to center? Investigating inertia with a multilevel autoregressive model. Front. Psychol. 5, 1492. https://doi.org/10.3389/fpsyg.2014.01492

Hollenstein, T., 2015. This Time, It's Real: Affective Flexibility, Time Scales, Feedback Loops, and the Regulation of Emotion. Emot. Rev. 7, 308-315. https://doi.org/10.1177/1754073915590621

Hollenstein, T., Lichtwarck-Aschoff, A., Potworowski, G., 2013. A Model of Socioemotional Flexibility at Three Time Scales. Emot. Rev. 5, 397-405. https://doi.org/10.1177/1754073913484181

Houben, M., Van Den Noortgate, W., Kuppens, P., 2015. The relation between short-term emotion dynamics and psychological well-being: A meta-analysis. Psychol. Bull. 141, 901930. https://doi.org/10.1037/a0038822

Husen, K., Rafaeli, E., Rubel, J.A., Bar-Kalifa, E., Lutz, W., 2016. Daily affect dynamics predict early response in CBT: Feasibility and predictive validity of EMA for outpatient psychotherapy. J. Affect. Disord. 206, 305-314. https://doi.org/10.1016/j.jad.2016.08.025

Ironside, M., Kumar, P., Kang, M.S., Pizzagalli, D.A., 2018. Brain mechanisms mediating effects of stress on reward sensitivity. Curr. Opin. Behav. Sci. 22, 106-113. https://doi.org/10.1016/j.cobeha.2018.01.016

Jahng, S., Wood, P.K., Trull, T.J., 2008. Analysis of Affective Instability in Ecological Momentary Assessment: Indices Using Successive Difference and Group Comparison via Multilevel Modeling. Psychol. Methods 13, 354-375. https://doi.org/10.1037/a0014173

Kercher, K., 1992. Assessing Subjective Well-Being in the Old-Old. Res. Aging 14, 131-168. 
https://doi.org/10.1177/0164027592142001

Kessler, R.C., Chiu, W.T., Demler, O., Walters, E.E., 2005. Prevalence, severity, and comorbidity of 12-month DSM-IV disorders in the National Comorbidity Survey Replication. Arch. Gen. Psychiatry 62, 617. https://doi.org/10.1001/archpsyc.62.6.617

Klein, D.N., Shankman, S.A., Lewinsohn, P.M., Rohde, P., Seeley, J.R., 2004. Family Study of Chronic Depression in a Community Sample of Young Adults. Am. J. Psychiatry 161, 646653. https://doi.org/10.1176/appi.ajp.161.4.646

Koval, P., Brose, A., Pe, M.L., Houben, M., Erbas, Y., Champagne, D., Kuppens, P., 2015a. Emotional inertia and external events: The roles of exposure, reactivity, and recovery. Emotion 15, 625-636. https://doi.org/10.1037/emo0000059

Koval, P., Butler, E.A., Hollenstein, T., Lanteigne, D., Kuppens, P., 2015b. Emotion regulation and the temporal dynamics of emotions: Effects of cognitive reappraisal and expressive suppression on emotional inertia. Cogn. Emot. 29, 831-851.

https://doi.org/10.1080/02699931.2014.948388

Kraemer, H.C., Kazdin, A.E., Offord, D.R., Kessler, R.C., Jensen, P.S., Kupfer, D.J., 1997. Coming to terms with the terms of risk. Arch. Gen. Psychiatry. https://doi.org/10.1001/archpsyc.1997.01830160065009

Kuppens, P., Sheeber, L.B., Yap, M.B.H., Whittle, S., Simmons, J.G., Allen, N.B., 2012. Emotional inertia prospectively predicts the onset of depressive disorder in adolescence. Emotion 12, 283-289. https://doi.org/10.1037/a0025046

Kuznetsova, A., Brockhoff, P.B., Christensen, R.H.B., 2017. lmerTest Package: Tests in Linear Mixed Effects Models. J. Stat. Softw. 82. https://doi.org/10.18637/jss.v082.i13

Meyer, A., Lerner, M.D., De Los Reyes, A., Laird, R.D., Hajcak, G., 2017. Considering ERP 
difference scores as individual difference measures: Issues with subtraction and alternative approaches. Psychophysiology 54, 114-122. https://doi.org/10.1111/psyp.12664

Nakagawa, S., Schielzeth, H., 2013. A general and simple method for obtaining R 2 from generalized linear mixed-effects models. Methods Ecol. Evol. 4, 133-142. https://doi.org/10.1111/j.2041-210x.2012.00261.x

Nelson, B.D., Perlman, G., Klein, D.N., Kotov, R., Hajcak, G., 2016. Blunted Neural Response to Rewards as a Prospective Predictor of the Development of Depression in Adolescent Girls. Am. J. Psychiatry 173, 1223-1230. https://doi.org/10.1176/appi.ajp.2016.15121524

Nelson, G.H., O'Hara, M.W., Watson, D., 2018. National norms for the expanded version of the inventory of depression and anxiety symptoms (IDAS-II). J. Clin. Psychol. 74, 953-968. https://doi.org/10.1002/jclp.22560

Nunnally, J., Bernstein, I., 1994. Psychometric Theory, 3rd ed. McGraw-Hill, New York, NY. Pemberton, R., Fuller Tyszkiewicz, M.D., 2016. Factors contributing to depressive mood states in everyday life: A systematic review. J. Affect. Disord. 200, 103-110. https://doi.org/10.1016/j.jad.2016.04.023

Perrin, F., Pernier, J., Bertrand, O., Echallier, J.F., 1989. Spherical splines for scalp potential and current density mapping. Electroencephalogr. Clin. Neurophysiol. 72, 184-187. https://doi.org/10.1016/0013-4694(89)90180-6

R Core Team, 2019. R: A Language and Environment for Statistical Computing.

Robinson, G.K., 1991. That BLUP is a Good Thing: The Estimation of Random Effects. Stat. Sci. 6, 15-32. https://doi.org/10.1214/ss/1177011933

Rottenberg, J., 2005. Mood and emotion in major depression. Curr. Dir. Psychol. Sci. 14, 167170. https://doi.org/10.1111/j.0963-7214.2005.00354.x 
Schoevers, R.A., van Borkulo, C.D., Lamers, F., Servaas, M.N., Bastiaansen, J.A., Beekman, A.T.F., van Hemert, A.M., Smit, J.H., Penninx, B.W.J.H., Riese, H., 2020. Affect fluctuations examined with ecological momentary assessment in patients with current or remitted depression and anxiety disorders. Psychol. Med. 1-10. https://doi.org/10.1017/S0033291720000689

Shankman, S.A., Funkhouser, C.J., Klein, D.N., Davila, J., Lerner, D., Hee, D., 2018. Reliability and validity of severity dimensions of psychopathology assessed using the Structured Clinical Interview for DSM-5 (SCID). Int. J. Methods Psychiatr. Res. 27, e1590. https://doi.org/10.1002/mpr.1590

Shankman, S.A., Klein, D.N., 2003. The relation between depression and anxiety: An evaluation of the tripartite, approach-withdrawal and valence-arousal models. Clin. Psychol. Rev. 23, 605-37. https://doi.org/10.1016/S0272-7358(03)00038-2

Solhan, M.B., Trull, T.J., Jahng, S., Wood, P.K., 2009. Clinical Assessment of Affective Instability: Comparing EMA Indices, Questionnaire Reports, and Retrospective Recall. Psychol. Assess. 21, 425-436. https://doi.org/10.1037/a0016869

Sperry, S.H., Walsh, M.A., Kwapil, T.R., 2020. Emotion dynamics concurrently and prospectively predict mood psychopathology. J. Affect. Disord. 261, 67-75. https://doi.org/10.1016/j.jad.2019.09.076

Stevens, E.S., Lieberman, L., Funkhouser, C.J., Correa, K.A., Shankman, S.A., 2019. Startle during threat longitudinally predicts functional impairment independent of DSM diagnoses. Psychiatry Res. 279, 207-215. https://doi.org/10.1016/j.psychres.2019.02.072

Trull, T.J., Lane, S.P., Koval, P., Ebner-Priemer, U.W., 2015. Affective Dynamics in Psychopathology. Emot. Rev. 7, 355-361. https://doi.org/10.1177/1754073915590617 
Tversky, A., Kahneman, D., 1992. Advances in prospect theory: Cumulative representation of uncertainty. J. Risk Uncertain. 5, 297-323. https://doi.org/10.1007/BF00122574

van de Leemput, I.A., Wichers, M., Cramer, A.O.J., Borsboom, D., Tuerlinckx, F., Kuppens, P., Van Nes, E.H., Viechtbauer, W., Giltay, E.J., Aggen, S.H., Derom, C., Jacobs, N., Kendler, K.S., Van Der Maas, H.L.J., Neale, M.C., Peeters, F., Thiery, E., Zachar, P., Scheffer, M., 2014. Critical slowing down as early warning for the onset and termination of depression. Proc. Natl. Acad. Sci. 111, 87-92. https://doi.org/10.1073/pnas.1312114110

Watson, D., Clark, L.A., Tellegen, A., 1988. Development and validation of brief measures of positive and negative affect: the PANAS scales. J. Pers. Soc. Psychol. 54, 1063-70.

Watson, D., O’Hara, M.W., Chmielewski, M., McDade-Montez, E.A., Koffel, E., Naragon, K., Stuart, S., 2008. Further Validation of the IDAS: Evidence of Convergent, Discriminant, Criterion, and Incremental Validity. Psychol. Assess. 20, 248-259. https://doi.org/10.1037/a0012570

Watson, D., O’Hara, M.W., Naragon-Gainey, K., Koffel, E., Chmielewski, M., Kotov, R., Stasik, S.M., Ruggero, C.J., 2012. Development and Validation of New Anxiety and Bipolar Symptom Scales for an Expanded Version of the IDAS (the IDAS-II). Assessment 19, 399420. https://doi.org/10.1177/1073191112449857

Watson, D., O’Hara, M.W., Simms, L.J., Kotov, R., Chmielewski, M., McDade-Montez, E.A., Gamez, W., Stuart, S., 2007. Development and validation of the Inventory of Depression and Anxiety Symptoms (IDAS). Psychol. Assess. 19, 253-268. https://doi.org/10.1037/1040-3590.19.3.253

Waugh, C.E., Shing, E.Z., Avery, B.M., Jung, Y., Whitlow, C.T., Maldjian, J.A., 2017. Neural predictors of emotional inertia in daily life. Soc. Cogn. Affect. Neurosci. 12, 1448-1459. 
https://doi.org/10.1093/scan/nsx071

Weinberg, A., Liu, H., Hajcak, G., Shankman, S.A., 2015. Blunted neural response to rewards as a vulnerability factor for depression: Results from a family study. J. Abnorm. Psychol. 124, 878-89. https://doi.org/10.1037/abn0000081

Weinberg, A., Liu, H., Shankman, S.A., 2016. Blunted neural response to errors as a trait marker of melancholic depression. Biol. Psychol. 113, 100-107. https://doi.org/10.1016/j.biopsycho.2015.11.012

Weinberg, A., Shankman, S.A., 2017. Blunted Reward Processing in Remitted Melancholic Depression. Clin. Psychol. Sci. 5, 14-25. https://doi.org/10.1177/2167702616633158

Weissman, M.M., Wickramaratne, P., Nomura, Y., Warner, V., Pilowsky, D., Verdeli, H., 2006. Offspring of depressed parents: 20 Years later. Am. J. Psychiatry 163, 1001-1008. https://doi.org/10.1176/ajp.2006.163.6.1001

Wichers, M.C., 2014. The dynamic nature of depression: A new micro-level perspective of mental disorder that meets current challenges. Psychol. Med. https://doi.org/10.1017/S0033291713001979

Wichers, M.C., Peeters, F., Geschwind, N., Jacobson, N.S., Simons, C.J.P., Derom, C., Thiery, E., Delespaul, P.H., Van Os, J., 2010. Unveiling patterns of affective responses in daily life may improve outcome prediction in depression: A momentary assessment study. J. Affect. Disord. 124, 191-195. https://doi.org/10.1016/j.jad.2009.11.010

Zeiss, A.M., Lewinsohn, P.M., 1988. Enduring deficits after remissions of depression: A test of the scar hypothesis. Behav. Res. Ther. 26, 151-158. https://doi.org/10.1016/00057967(88)90114-3 
Table 1. Demographic and clinical characteristics of the sample.

\begin{tabular}{lc}
\hline Characteristic & Frequency $(\%)$ or Mean (SD) \\
\hline Age $(M, S D)$ & $22.08(3.20)$ \\
Sex = Female (\%) & $43(72.9)$ \\
Ethnicity (\%) & \\
Caucasian & $22(37.3)$ \\
African American & $9(15.3)$ \\
Hispanic & $15(25.4)$ \\
Asian & $9(15.3)$ \\
Mixed Race or Other & $4(6.8)$ \\
Education (\%) & \\
Some high school & $1(1.7)$ \\
Graduated high school or equivalent & $6(10.2)$ \\
Some college & $27(45.8)$ \\
Graduated 2-year college & $5(8.5)$ \\
Graduated 4-year college & $20(33.9)$ \\
Personal MDD History (\%) & $30(50.8)$ \\
Family MDD History (\%) & $26(44.8)$ \\
Current Diagnoses (\%) & \\
Alcohol Use Disorder & $0(0.0)$ \\
Substance Use Disorder & $0(0.0)$ \\
Posttraumatic Stress Disorder & $2(3.4)$ \\
Panic Disorder & $0(0.0)$ \\
Agoraphobia & $0(0.0)$ \\
Social Anxiety Disorder & $0(0.0)$ \\
Specific Phobia & $2(3.4)$ \\
Generalized Anxiety Disorder & $0(0.0)$ \\
Obsessive Compulsive Disorder & $0(0.0)$ \\
\hline
\end{tabular}

Note. MDD = Major Depressive Disorder. 
Table 2. Relationships between depression risk factors and mean levels, instability, and inertia of positive and negative affect.

\begin{tabular}{lcccccc}
\hline & \multicolumn{2}{c}{ Positive Affect } & & \multicolumn{3}{c}{ Negative Affect } \\
Risk Factor & Mean & Instability & Inertia & Mean & Instability & Inertia \\
\hline MDD History & $-.13[-.49, .26]$ & $.01[-.15, .17]$ & $-.01[-.16, .11]$ & $\mathbf{. 4 2 *}[.05, .41]$ & $-.12[-.22, .02]$ & $.08[-.07, .27]$ \\
MDD Family History & $.31[-.07, .59]$ & $-.01[-.15, .14]$ &. $\mathbf{1 0 *}[. \mathbf{0 3}, .27]$ & $-.07[-.21, .13]$ & $-.02[-.11, .08]$ & $-.10[-.29, .06]$ \\
Reward Positivity & $-.01[-.18, .16]$ & $.00[-.07, .07]$ & $.02[-.03, .11]$ & $-.13[-.15, .01]$ & $-.01[-.05, .04]$ & $\mathbf{- . 0 8 *}[-.20, .00]$ \\
\hline
\end{tabular}

Note. ${ }^{*} p<.05$

Coefficients are presented as standardized betas. Brackets contain 95\% confidence intervals. All models covary for current depressive symptoms as measured by the IDAS General Depression scale. Significant effects $(p<.05)$ are bolded. $\mathrm{MDD}=$ Major Depressive Disorder. 

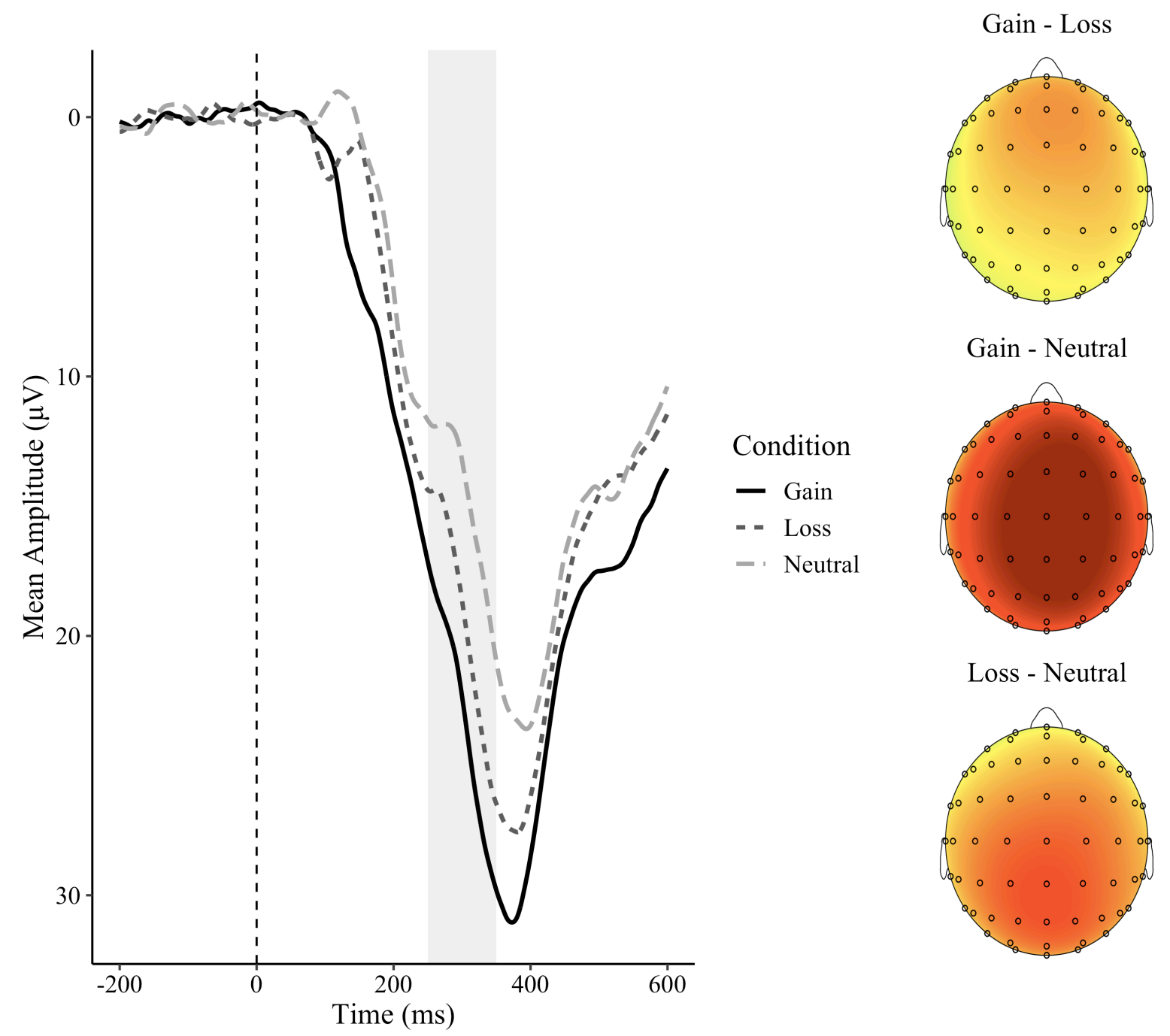

Gain - Neutral
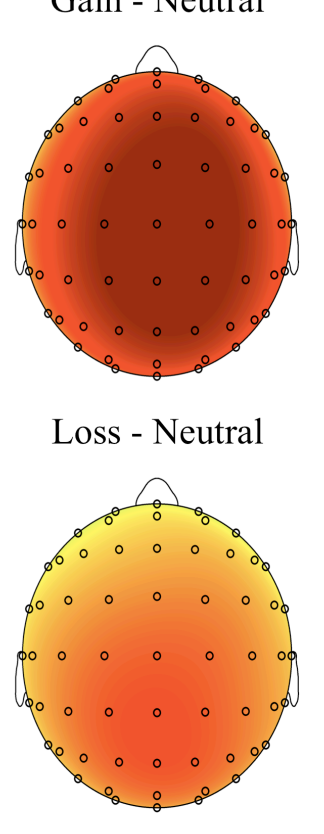

Figure 1. (A) Waveforms representing mean amplitudes to each condition. (B) Topographical maps representing differences in reactivity between conditions. 


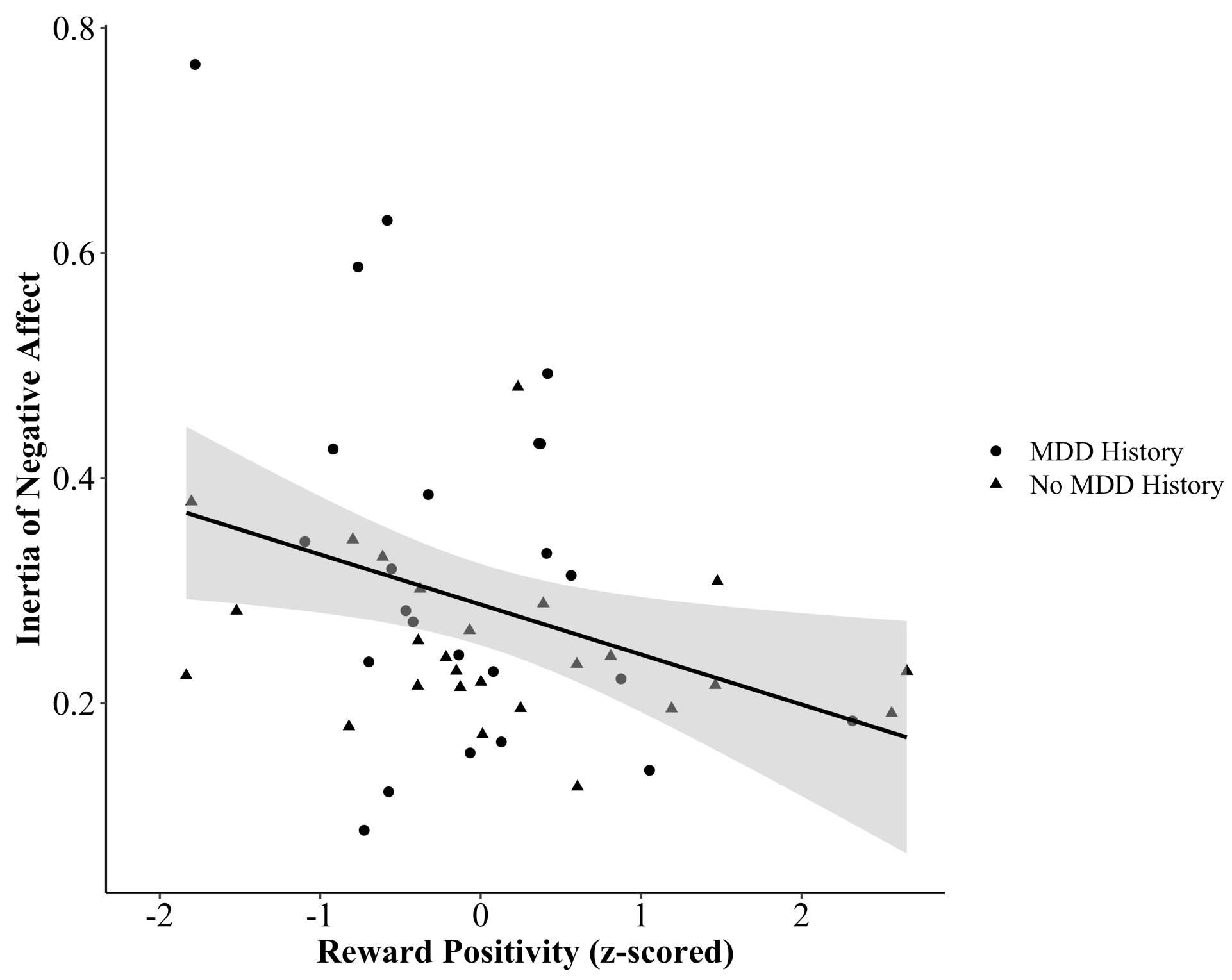

Figure 2. The relationship between the Reward Positivity and best linear unbiased predictions (BLUPs) of the inertia of negative affect (i.e., random autoregressive slope of negative affect at time $t$ predicting negative affect at time $t+1$ ). 
A

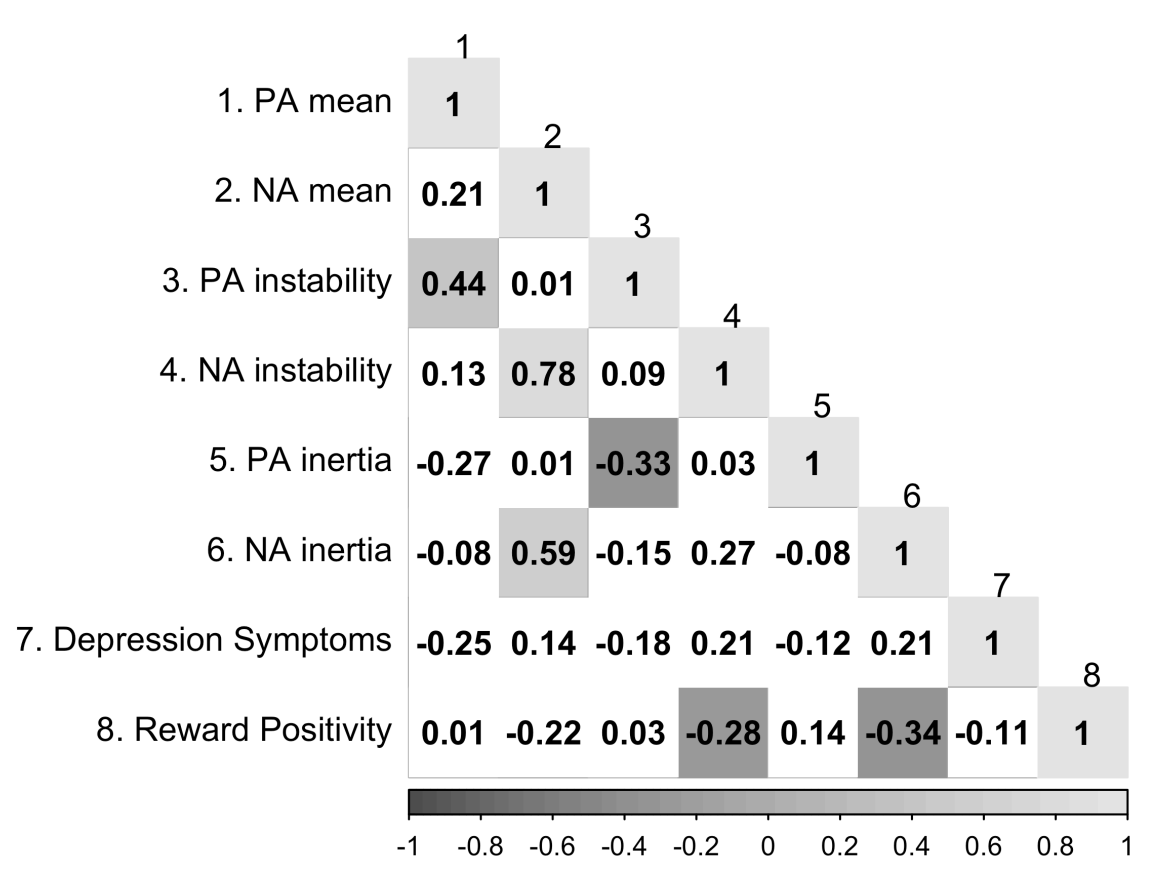

B

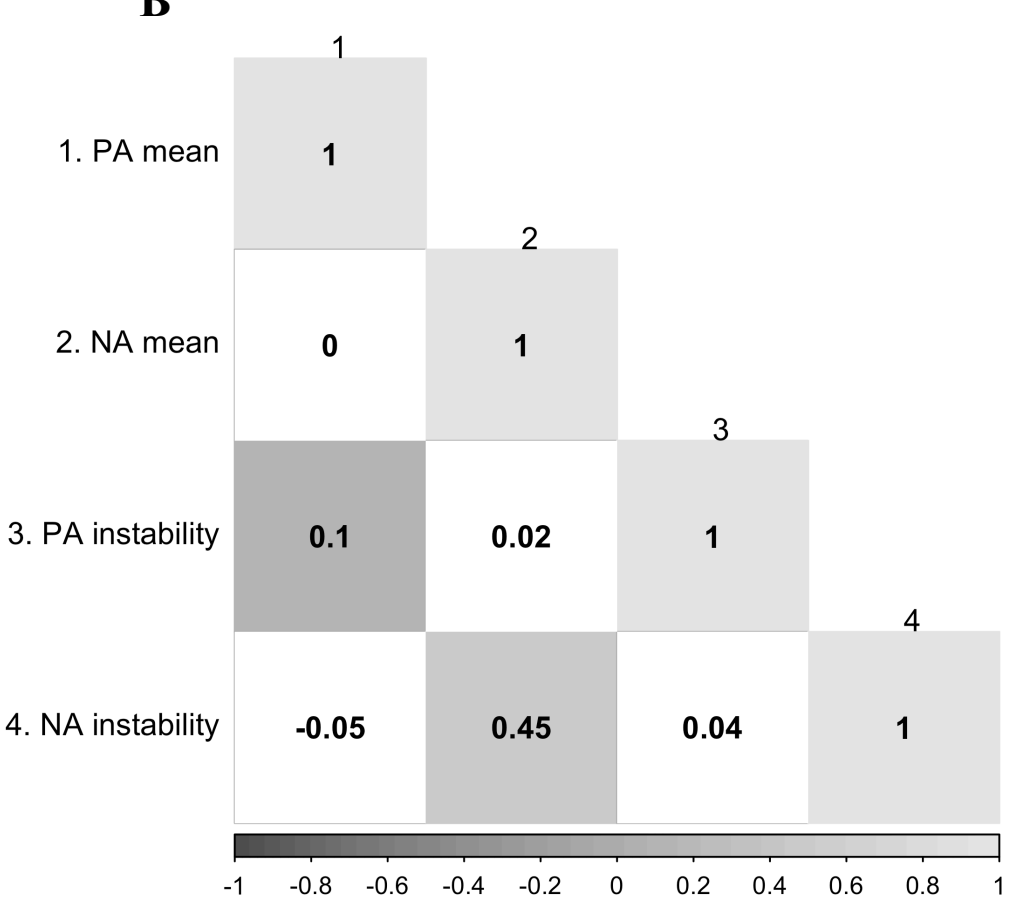

Figure 3. (A) Correlations between continuous study variables. As instability and inertia were computed at the within-person level, multilevel models were used to generate best linear unbiased predictions (BLUPs) for each participant's instability and inertia of positive and negative affect. (B) Within-subject correlations between affect dynamics indices. Significant correlations $(p<.05)$ are shaded, and the degree of shading corresponds to the strength of the correlation. 